\title{
LISTA DE TERMOS DA LINGUÍSTICA SISTÊMICO-FUNCIONAL EM PORTUGUÊS BRASILEIRO: LÉXICO-GRAMÁTICA
}

\section{LIST OF TERMS OF SYSTEMIC FUNCTIONAL LINGUISTICS IN BRAZILIAN PORTUGUESE: LEXICOGRAMMAR}

\author{
Sara Regina Scotta Cabral (UFSM) \\ sara.scotta.cabral@gmail.com \\ Orlando Vian Jr. (UNIFESP/CNPq) \\ vian.junior@unifesp.br \\ Cristiane Fuzer (UFSM) \\ cristiane.fuzer@ufsm.br \\ Lucia Rottava (UFRGS) \\ luciarottava@yahoo.com.br \\ Karen Andresa Teixeira Santorum (UFSM) \\ karensantorum13@gmail.com
}

\section{Apresentação}

Neste número temático da Revista Organon da UFRGS, oferecemos aos leitores uma Lista de Termos da Linguística Sistêmico-Funcional em Português Brasileiro no que se refere ao estrato léxico-gramatical da teoria hallidayana. Essa lista representa uma contribuição dos organizadores deste número temático conjuntamente com representantes do Grupo SAL Sistêmica, Ambientes e Linguagens, cadastrado no CNPq, e do Grupo de Trabalho de Linguística Sistêmico-Funcional da ANPOLL - Associação Nacional de Pós-Graduação e Pesquisa em Letras e Linguística.

A finalidade da Lista de Termos é divulgar e ampliar a Linguística SistêmicoFuncional LSF no contexto brasileiro para que, além de pesquisadores e pós-graduandos, alunos da graduação e interessados em linguagem possam ter acesso a essa teoria e ao uso dos termos mais recorrentes. Trata-se de indicações e de sugestões que estão abertas ao debate e que podem ser retroalimentadas com pesquisas em andamento ou com estudos futuros. Afinal, 
a linguagem em funcionamento está sujeita a novos contornos de padrões de significação, e uma teoria deve ser sensível a essas mudanças e desdobramentos.

A elaboração dessa Lista de Termos foi uma tarefa com múltiplas contribuições. A origem está na Lista de Termos da PUC-SP produzida sob coordenação da Profa. Dra. Leila Barbara e do Prof. Dr. Carlos Gouveia em um grupo que reunia orientandos de mestrado e de doutorado da professora Leila. Nesse período, o Programa de Linguística Aplicada e Estudos da Linguagem (LAEL) da PUC-SP mantinha um projeto bilateral com a Universidade de Lisboa. A lista tinha a intenção de atender às variantes europeia e brasileira. Constava de 265 termos e era baseada na segunda edição da gramática de Halliday (1994) e se intitula "Termos de gramática sistémico-funcional em português", aprovados para utilização pelos participantes na lista de discussão.

À Lista que ora apresentamos foram acrescentados termos advindos das edições posteriores da Gramática Sistêmico-Funcional de Halliday e Matthiessen (2004; 2014). Atualizações e ajustes são provenientes das publicações de Fuzer e Cabral (2014) e de Cunha e Souza (2011), de inúmeras dissertações e teses produzidas nos diferentes programas de pósgraduação em contexto brasileiro, e de capítulos de livros e artigos publicados que abordam a léxico-gramática sob o viés da LSF. Alguns termos iniciam com maiúscula por convenção da teoria, para distinguir funções léxico-gramaticais. Por conter termos específicos da teoria sistêmico-funcional, a presente Lista não veicula termos característicos da gramática tradicional, a exemplo de adjetivo, advérbio, sujeito, etc.

Ressaltamos que a Lista é parte de uma proposta mais ampla que está sendo desenvolvida pela Associação de Linguística Sistêmico-Funcional da América Latina (ALSFAL), a qual visa elaborar um Glossário Trilíngue (inglês, espanhol e português), contemplando outros estratos e aspectos relacionados à LSF.

\section{LISTA DE TERMOS DA LINGUÍSTICA SISTÊMICO-FUNCIONAL EM PORTUGUÊS BRASILEIRO: LÉXICO-GRAMÁTICA}

\begin{tabular}{|r||l|}
\hline \multicolumn{1}{|c|}{ inglês } & \multicolumn{1}{|c|}{ português } \\
\hline ability [modality] & A \\
\hline Accompaniment [circumstance] & habilidade [modalidade] \\
\hline act clause & Acompanhamento [circunstância] \\
\hline active [voice] & oração-ato \\
\hline Actor & ativa [voz] \\
\hline addition [logico-semantic relation] & Ator \\
\hline
\end{tabular}




\begin{tabular}{|c|c|}
\hline Additive & Aditivo(a) \\
\hline adjectival group & grupo adjetivo \\
\hline Adjunct & Adjunto \\
\hline adverbial group & grupo adverbial \\
\hline adversative [logico-semantic relation] & adversativa [relação lógico-semântica] \\
\hline affective [mental process] & afetivo [processo mental] \\
\hline agency & agência \\
\hline Agent [participant] & Agente [participante] \\
\hline agentive & agentivo(a) \\
\hline agnate & agnato(a) \\
\hline agnation & agnação \\
\hline agreement & concordância \\
\hline alternation [logico-semantic relation] & alternância [relação lógico-semântica] \\
\hline alternative & alternativo(a) \\
\hline Angle [circumstance] & Ângulo [circunstância] \\
\hline angle [circumstantial clause] & ângulo [oração circunstancial] \\
\hline ascribe & inscrever \\
\hline ascriptive & inscritivo(a) \\
\hline asseverative & asseverativo(a) \\
\hline Assigner & Designador \\
\hline Attribute & Atributo \\
\hline attributive clause & oração atributiva \\
\hline attributive process & processo atributivo \\
\hline Attributor & Atribuidor \\
\hline \multicolumn{2}{|c|}{ B } \\
\hline Behalf [circumstance] & Benefício [circunstância] \\
\hline Behaver [participant] & Comportante [participante] \\
\hline Behaviour & Comportamento \\
\hline behavioural clause & oração comportamental \\
\hline behavioural process & processo comportamental \\
\hline Beneficiary & Beneficiário \\
\hline $\begin{array}{r}\text { bidirectionality [property of the mental } \\
\text { processes of emotion] }\end{array}$ & $\begin{array}{l}\text { bidirecionalidade [propriedade dos } \\
\text { processos mentais de emoção] }\end{array}$ \\
\hline binder & conector nas orações hipotáticas \\
\hline bound & preso(a) \\
\hline bracketing & agrupamento \\
\hline \multicolumn{2}{|c|}{$\mathbf{C}$} \\
\hline Carrier & Portador \\
\hline case [noun of fact] & acontecimento [nome de um fato] \\
\hline Cause & Causa \\
\hline Cause Adjunct & Adjunto de Causa \\
\hline certainty [modality] & certeza [modalidade] \\
\hline chance [fact noun] & possibilidade [nome factual] \\
\hline Circumstance & Circunstância \\
\hline circumstance [modulation] & circunstância [modulação] \\
\hline circumstance [transitivity role] & circunstância [papel de transitividade] \\
\hline circumstance type [adverbial group] & tipo de circunstância [grupo adverbial] \\
\hline circumstantial relational clause & oração relacional circunstancial \\
\hline
\end{tabular}




\begin{tabular}{|c|c|}
\hline circumstantial Adjunct & Adjunto circunstancial \\
\hline circumstantial Attribute & Atributo circunstancial \\
\hline circumstantial clause & oração circunstancial \\
\hline $\begin{array}{r}\text { circumstantiation [circumstantial } \\
\text { transitivity] }\end{array}$ & $\begin{array}{l}\text { circunstancialização [transitividade } \\
\text { circunstancial] }\end{array}$ \\
\hline $\begin{array}{r}\text { clarification [elaborating logico-semantic } \\
\text { relation] }\end{array}$ & $\begin{array}{l}\text { clarificação [relação lógico-semântica de } \\
\text { elaboração] }\end{array}$ \\
\hline class [of grammatical unit] & classe [de unidade gramatical] \\
\hline Classifier & Classificador \\
\hline class-membership & membro de classe \\
\hline clause & oração \\
\hline clause as exchange & oração como troca \\
\hline clause as message & oração como mensagem \\
\hline clause as representation & oração como representação \\
\hline clause complex & complexo oracional \\
\hline clause nexus & nexo oracional \\
\hline cleft clause & oração clivada \\
\hline Client & Cliente \\
\hline cline & contínuo \\
\hline cline of delicacy & contínuo de detalhamento \\
\hline cluster & agrupamento \\
\hline cognitive [mental process] & cognitivo [processo mental] \\
\hline comitative & comitativo(a) \\
\hline command [speech function] & comando [função de fala] \\
\hline comment Adjunct & Adjunto de comentário \\
\hline commodity [speech function system] & mercadoria [sistema de função de fala] \\
\hline common noun & nome comum \\
\hline common verb & verbo comum \\
\hline comparative conjunction & conjunção comparativa \\
\hline comparison [logico-semantic relation] & comparação [relação lógico-semântica] \\
\hline Complement [modal function] & Complemento [função modal] \\
\hline complementarity & complementaridade \\
\hline complex clause & complexo oracional \\
\hline compound noun & |nome composto \\
\hline conation [hypotactic verbal group complex] & conação [grupo verbal complexo hipotático] \\
\hline Concession [circumstance] & |Concessão [circunstância] \\
\hline concession [enhancing relation] & concessão [relação de intensificação] \\
\hline concessive [logico-semantic relation] & concessivo [relação lógico-semântica] \\
\hline Condition [circumstance] & Condição [circunstância] \\
\hline condition [enhancing relation] & condição [relação de intensificação] \\
\hline conflation & confluência \\
\hline conflate & confluir \\
\hline congruence & | congruência \\
\hline congruent & congruente \\
\hline conjunction [word class] & conjunção [classe de palavra] \\
\hline conjunction group & grupo conjuntivo \\
\hline conjunctive [word class] & conjunção [classe de palavras] \\
\hline conjunctive Adjunct & Adjunto conjuntivo \\
\hline
\end{tabular}




\begin{tabular}{|c|c|}
\hline conjunctive marker & marcador conjuntivo \\
\hline consequence [logico-semantic relation] & consequência [relação lógico-semântica] \\
\hline Contingency [circumstance] & Contingência [circunstância] \\
\hline continuative & continuativo(a) \\
\hline copular verb & verbo copulativo \\
\hline correlative [conjunction] & correlativa [conjunção] \\
\hline count noun & nome contável \\
\hline coupling & acoplamento \\
\hline creative [material clause] & criativa [oração material] \\
\hline \multicolumn{2}{|l|}{ D } \\
\hline declarative clause & oração declarativa \\
\hline declarative [mood] & declarativo [modo] \\
\hline Default [circumstance] & Falta [circunstância] \\
\hline defining relative clause & oração relativa definidora \\
\hline Degree [circunstância] & Grau [circunstância] \\
\hline Deictic & Dêitico \\
\hline deicticity & deiticidade \\
\hline deixis & dêixis \\
\hline delicacy & detalhamento \\
\hline demanding & solicitação \\
\hline determiner & determinante \\
\hline demonstrative & demonstrativo \\
\hline deontic [modality] & deôntica [modalidade] \\
\hline dependent clause & oração dependente \\
\hline depictive & depictivo(a) \\
\hline describe, description & descrever, descrição \\
\hline desiderative [type of sensing] & desiderativo [tipo de sentimento] \\
\hline determiner [nominal word class] & determinante [classe de palavra nominal] \\
\hline direct speech & discurso direto \\
\hline discourse Adjunct & Adjunto discursivo \\
\hline doing [field of activity] & fazer [campo de atividade] \\
\hline dominant clause & oração dominante \\
\hline downranked [clause] & rebaixada [oração] \\
\hline downranking & rebaixamento \\
\hline duration/inception [phase] & duração/incepção [fase] \\
\hline dynamic [modality] & dinâmica [modalidade] \\
\hline \multicolumn{2}{|c|}{$\mathbf{E}$} \\
\hline effective [agency] & efetiva [agência] \\
\hline elaboration [logico-semantic relation] & elaboração [relação lógico-semântica] \\
\hline element & elemento \\
\hline embedded & encaixado(a) \\
\hline embedded clause & oração encaixada \\
\hline embedded expansion & expansão encaixada \\
\hline embedding & encaixamento \\
\hline emotive & emotivo(a) \\
\hline engendered & causado \\
\hline enhancement [logico-semantic relation] & intensificação [relação lógico-semântica] \\
\hline entity & entidade \\
\hline
\end{tabular}




\begin{tabular}{|c|c|}
\hline Epithet & Epíteto \\
\hline equative & equativo(a) \\
\hline equivalence [identifying clause] & equivalência [oração identificativa] \\
\hline ergative model & modelo ergativo \\
\hline ergative/non-ergative pairs & pares ergativos/não ergativos \\
\hline ergativity & ergatividade \\
\hline Event [verbal group function] & Evento [função do grupo verbal] \\
\hline exchange [move] & troca [movimento] \\
\hline exchange process & processo de troca \\
\hline exclamative [mood] & exclamativo [modo] \\
\hline exemplification [logico-semantic relation] & exemplificação [relação lógico-semântica] \\
\hline Existent & Existente \\
\hline existential clause & oração existencial \\
\hline existencial process & processo existencial \\
\hline expansion & expansão \\
\hline expansion as logical-semantic relationship & expansão como relação lógico-semântica \\
\hline explaining & explicar \\
\hline Expletive & Expletivo \\
\hline explicit & explícito(a) \\
\hline explicit/implicit orientation & orientação explícita/implícita \\
\hline exposition [logico-semantic relation] & exposição [relação lógico-semântica] \\
\hline extension [logico-semantic relation] & extensão [relação lógico-semântica] \\
\hline Extent [circumstance] & Extensão [circunstância] \\
\hline \multicolumn{2}{|c|}{$\mathbf{F}$} \\
\hline fact clause & oração-fato \\
\hline figure & figura \\
\hline Finite & Finito \\
\hline finite [clause] & finita [oração] \\
\hline finite operator & operador finito \\
\hline finite verbal group & grupo verbal finito \\
\hline finite verbal operator & operador verbal finito \\
\hline flow & fluxo \\
\hline Frequency [circumstance] & Frequência [circunstância] \\
\hline frequency [modulation] & frequência [modulação] \\
\hline function [stratification matrix] & função [matriz de estratificação] \\
\hline \multicolumn{2}{|c|}{$\mathbf{G}$} \\
\hline Given [information unit function] & Dado [função de unidade informacional] \\
\hline giving/demanding [speech function] & dar/solicitar [função de fala] \\
\hline Goal & Meta \\
\hline goods-\&-services/ information & bens e serviços / informação \\
\hline grammatical intricacy & intricacia gramatical \\
\hline grammatical metaphor & metáfora gramatical \\
\hline grammaticalization & gramaticalização \\
\hline grammatical Subject & Sujeito gramatical \\
\hline grammatics & gramaticologia [teoria gramatical] \\
\hline greeting [minor clause] & saudação [oração menor] \\
\hline group & grupo \\
\hline group function & função de grupo \\
\hline
\end{tabular}




\begin{tabular}{|c|c|}
\hline group complex & complexo de grupo \\
\hline Guise [circumstance] & Aparência [circunstância] \\
\hline \multicolumn{2}{|c|}{ H } \\
\hline Head [group function] & Núcleo [função no grupo] \\
\hline hyper-New & hiper-Novo \\
\hline hyperphenomenal & hiperfenomenal \\
\hline hyper-Theme & hiper-Tema \\
\hline hypotatic & hipotático(a) \\
\hline hypotaxis & hipotaxe \\
\hline \multicolumn{2}{|c|}{ I } \\
\hline Idea & Ideia \\
\hline idea clause & oração ideia \\
\hline ideational metaphor & metáfora ideacional \\
\hline Identified & Identificado \\
\hline Identifier & Identificador \\
\hline identifying clause & oração identificativa \\
\hline identifying process & processo identificativo \\
\hline imperative [mood] & imperativo [modo] \\
\hline imperfective/perfective [aspect] & imperfectivo/perfectivo [aspecto] \\
\hline implicit & implícito(a) \\
\hline inceptivo [attribution clause] & inceptivo [oração atributiva] \\
\hline inclination [modality] & inclinação [modalidade] \\
\hline incongruent [realization] & incongruente [realização] \\
\hline independent clause & oração independente \\
\hline indicative [mood] & indicativo [modo] \\
\hline indirect question & pergunta indireta \\
\hline indirect speech & discurso indireto \\
\hline indirect statements/questions & afirmações/perguntas indiretas \\
\hline Inducer [participant] & Indutor [participante] \\
\hline information focus & foco de informação \\
\hline information unit & unidade de informação \\
\hline Initiator [participant] & Iniciador [participante] \\
\hline intensification & intensificação \\
\hline intensifier & intensificador \\
\hline intensity & intensidade \\
\hline intensive & intensivo(a) \\
\hline intensive attributive clause & oração atributiva intensiva \\
\hline intensive clause & oração intensiva \\
\hline intensive identifying clause & oração identificativa intensiva \\
\hline interdependency [taxis] & interdependência [taxe] \\
\hline interpersonal Adjunct & Adjunto interpessoal \\
\hline interpersonal metaphor & metáfora interpessoal \\
\hline interpersonal projection & projeção interpessoal \\
\hline interpersonal Theme & Tema interpessoal \\
\hline interrogative [mood] & \begin{tabular}{|l} 
interrogativo [modo] \\
\end{tabular} \\
\hline interval [circumstance of extent] & intervalo [circunstância de extensão] \\
\hline \multicolumn{2}{|c|}{ 2 } \\
\hline jussive [imperative] & jussivo(a) [imperativo] \\
\hline
\end{tabular}


$\mathbf{L}$

lexical density

lexical ergativity

lexical grammar

lexical verb [word class] lexicogrammar

lexis

like/please, mental clause

linker

Location [circumstance]

locative demonstrative [word class] locution

logical function

logical metafunction logical mode

logical relation

logical Subject

logico-semantic type

logico-semantic relation

M

\begin{tabular}{|c|c|}
\hline macrophenomenon & macrofenômeno \\
\hline major clause & oração maior \\
\hline Manner [circumstance] & Modo [circunstância] \\
\hline manner [logico-semantic relation] & modo [relação lógico-semântica] \\
\hline marked Theme & Tema marcado \\
\hline mass noun & nome de massa \\
\hline material clause & oração material \\
\hline material process & processo material \\
\hline matrix & matriz \\
\hline Matter [circumstance] & Assunto [circunstância] \\
\hline mean [logico-semantic relation] & meio [relação lógico-semântica] \\
\hline meaning & significado \\
\hline Means [circumstance] & Meio [circunstância] \\
\hline Medium [role in ergative model] & Mediador [papel no modelo ergativo] \\
\hline mental clause & oração mental \\
\hline mental process & processo mental \\
\hline message & mensagem \\
\hline metaphor & metáfora \\
\hline metaphorical & metafórico(a) \\
\hline meteorological clause & oração meteorológica \\
\hline middle [agency] & média [agência] \\
\hline middle voice & voz média \\
\hline minor clause & oração menor \\
\hline minor Process & Processo menor \\
\hline modal Adjunct & Adjunto modal \\
\hline modal assessment & avaliação modal \\
\hline modal operator & operador modal \\
\hline modality & modalidade \\
\hline
\end{tabular}




\begin{tabular}{|c|c|}
\hline modalization & modalização \\
\hline modal value & valor modal \\
\hline mode [contextual parameter] & modo [parâmetro contextual] \\
\hline mode [verbal category] & modo [categoria verbal] \\
\hline Modifier [in the group] & Modificador [no grupo] \\
\hline modifier & modificador \\
\hline modulation & modulação \\
\hline MOOD system & sistema de MODO \\
\hline Mood [verbal] & Modo [verbal] \\
\hline mood & modo \\
\hline mood Adjunct & Adjunto de modo \\
\hline \multicolumn{2}{|c|}{$\mathbf{N}$} \\
\hline need [noun of modulation] & necessidade [nome de modulação] \\
\hline nesting & aninhamento \\
\hline New [information unit function] & Novo [função unidade de informação] \\
\hline nexus & nexo \\
\hline nominal clause & oração nominal \\
\hline nominal group & grupo nominal \\
\hline nominalization & nominalização \\
\hline non-agentive & não agentivo \\
\hline non-defining relative clause & oração relativa não definidora \\
\hline non-finite & não finito \\
\hline non-finite clause & oração não finita \\
\hline non-interactant & não interactante \\
\hline non-marked Theme & Tema não marcado \\
\hline non-projecting & não projetante \\
\hline noun [word class] & nome [classe de palavra] \\
\hline nucleus, clause & núcleo, oração \\
\hline Numerative [nominal group function] & Numerativo [função de grupo nominal] \\
\hline \multicolumn{2}{|c|}{$\mathbf{O}$} \\
\hline obligation [modulation] & obrigação [modulação] \\
\hline offer [speech function] & oferta [função de fala] \\
\hline operative/receptive [voice] & operativa/receptiva [voz] \\
\hline order of elements & ordem dos elementos \\
\hline Ordinative [nominal group function] & Ordinativo [função de grupo nominal] \\
\hline orientation & orientação \\
\hline \multicolumn{2}{|c|}{$\mathbf{P}$} \\
\hline paratatic & paratático(a) \\
\hline parataxis & parataxe \\
\hline participant [transitivity function] & participante [função de transitividade] \\
\hline passive [voice] & passiva [voz] \\
\hline patient [as term for participant] & paciente [como termo para participante] \\
\hline perception & percepção \\
\hline perceptive clause & oração perceptiva \\
\hline perfective/imperfective [aspect] & perfectivo/imperfectivo [aspecto] \\
\hline phase & fase \\
\hline Phenomenon & Fenômeno \\
\hline phrase & sintagma \\
\hline
\end{tabular}




\begin{tabular}{|c|c|}
\hline Place [circumstance] & Lugar [circunstância] \\
\hline place [logico-semantic relation] & lugar [relação lógico-semântica] \\
\hline point of departure [of the clause] & ponto de partida [da oração] \\
\hline polarity & polaridade \\
\hline possession as participant & posse como participante \\
\hline possessive & possessivo(a) \\
\hline possessive clause & oração possessiva \\
\hline post-Deictic & Pós-Dêitico \\
\hline Postmodifier & Pós-Modificador \\
\hline post-posed Subject & \begin{tabular}{|l|} 
Sujeito posposto \\
\end{tabular} \\
\hline predicate & predicado \\
\hline Predicate & Predicado \\
\hline Predicator & Predicador \\
\hline Premodifier & Pré-Modificador \\
\hline preposition group & grupo preposicional \\
\hline present-in-present [tense] & presente-no-presente [tempo] \\
\hline primary/secondary clause & oração primária/secundária \\
\hline primary/secondary group & grupo primário/secundário \\
\hline primary/secondary tense & tempo primário/secundário \\
\hline probability [modality] & probabilidade [modalidade] \\
\hline procedure & procedimento \\
\hline process type & tipo de processo \\
\hline Product [circumstance] & Produto [circunstância] \\
\hline progressive & progressivo(a) \\
\hline projection & projeção \\
\hline projected clause & oração projetada \\
\hline projecting clause & oração projetante \\
\hline proof [noun of indication] & prova [nome de indicação] \\
\hline proposal & proposta \\
\hline proposition & proposição \\
\hline $\begin{array}{r}\text { pseudo-cleft sentence [use of term in formal } \\
\text { grammar] }\end{array}$ & $\begin{array}{l}\text { oração pseudoclivada [uso do termo na } \\
\text { gramática formal] }\end{array}$ \\
\hline psychological Subject & Sujeito psicológico \\
\hline Purpose [circumstance] & Propósito [circunstância] \\
\hline purpose [logico-semantic relation] & propósito [relação lógico-semântica] \\
\hline \multicolumn{2}{|l|}{ Q } \\
\hline Qualifier [nominal group function] & Qualificador [função de grupo nominal] \\
\hline Quality [circumstance] & Qualidade [circunstância] \\
\hline quality [logico-semantic relation] & qualidade [relação lógico-semântica] \\
\hline $\begin{array}{r}\text { Quantitative } \\
\text { [nominal } \\
\text { group } \\
\text { function] }\end{array}$ & Quantitativa [função de grupo nominal] \\
\hline question [speech function] & pergunta [função de fala] \\
\hline quoting & citação \\
\hline \multicolumn{2}{|c|}{$\mathbf{R}$} \\
\hline Range [participant] & Alcance [participante] \\
\hline rank & nível \\
\hline
\end{tabular}




\begin{tabular}{|c|c|}
\hline rank scale & escala de nível \\
\hline rankshift; rank-shift & mudança de nível \\
\hline readiness [modality] & prontidão [modalidade] \\
\hline Reason [circumstance] & Razão [circunstância] \\
\hline reason [logico-semantic relation] & razão [relação lógico-semântica] \\
\hline Receiver [participant] & Receptor [participante de oração verbal] \\
\hline receptive & receptivo(a) \\
\hline receptive clause & oração receptiva \\
\hline Recipient [participant] & Recebedor [participante de oração material] \\
\hline relational clause & oração relacional \\
\hline relative clause & oração relativa \\
\hline relational process & processo relacional \\
\hline replacement [logico-semantic relation] & substituição [relação lógico-semântica] \\
\hline report & relato \\
\hline Residue & Resíduo \\
\hline response & resposta \\
\hline result [logico-semantic relation] & resultado [relação lógico-semântica] \\
\hline resultative attribute & atributo resultativo \\
\hline reversibility & reversibilidade \\
\hline Rheme & Rema \\
\hline Role [circumstance] & Papel [circunstância] \\
\hline root [modality] & raiz [modalidade] \\
\hline \multicolumn{2}{|c|}{$\mathbf{S}$} \\
\hline salience & saliência \\
\hline Sayer [participant] & Dizente [participante] \\
\hline Scope & Escopo \\
\hline secondary tense & tempo secundário \\
\hline semantic indeterminancy & indeterminação semântica \\
\hline Senser [participant] & Experienciador [participante] \\
\hline sentence [graphological unit] & sentença [unidade grafológica] \\
\hline simple clause & oração simples \\
\hline spatial [logico-semantic relation] & espacial [relação lógico-semântica] \\
\hline spatio-temporal enhancement & intensificação espaço-temporal \\
\hline speech role & papel de fala \\
\hline statement [speech function] & declaração [função de fala] \\
\hline stratum & estrato \\
\hline structural function & função estrutural \\
\hline structural Theme & Tema estrutural \\
\hline Subject [modal function] & Sujeito [função modal] \\
\hline subtraction [logico-semantic relation] & subtração [relação lógico-semântica] \\
\hline \multicolumn{2}{|c|}{$\mathbf{T}$} \\
\hline tactic relations & relações táticas \\
\hline Target [participant] & Alvo [participante da oração verbal] \\
\hline taxis & taxe \\
\hline temporal Adjunct & Adjunto de tempo \\
\hline temporal sequencing & sequenciamento temporal \\
\hline tenor [contextual parameter] & relações [parâmetro contextual] \\
\hline textual Adjunct & Adjunto textual \\
\hline
\end{tabular}




\begin{tabular}{|c|c|}
\hline textual Theme & Tema textual \\
\hline thematic category & categoria temática \\
\hline thematic dependent clause & oração dependente temática \\
\hline thematic equative & equativa temática \\
\hline Theme [textual clause function] & Tema [função oracional textual] \\
\hline theme predication & predicação de Tema \\
\hline Theme selection & seleção de Tema \\
\hline Thing [grammatical] & Ente [gramatical] \\
\hline thing[ phenomenon] & ser [fenômeno] \\
\hline Time [circumstance] & Tempo [circunstância] \\
\hline Token [participant] & Símbolo [participante] \\
\hline topical Theme & Tema tópico \\
\hline Topic-Comment & Tópico-Comentário \\
\hline transferred & transferido(a) \\
\hline transformative & transformativo(a) \\
\hline transitive model & modelo transitivo \\
\hline transitivity [clause system] & transitividade [sistema oracional] \\
\hline \multicolumn{2}{|c|}{$\mathbf{U}$} \\
\hline unmarked [type of Theme] & não marcado [tipo de Tema] \\
\hline usuality & usualidade \\
\hline utterance & enunciado \\
\hline \multicolumn{2}{|c|}{$\mathbf{V}$} \\
\hline Value & Valor \\
\hline value [modality: high, median, low] & valor [modalidade: alta, média, baixa] \\
\hline variation [logico-semantic relation] & variação [relação lógico-semântica] \\
\hline verbal clause & oração verbal \\
\hline verbal group & grupo verbal \\
\hline verbal operator & operador verbal \\
\hline verbal process & processo verbal \\
\hline Verbiage [participant] & Verbiagem [participante] \\
\hline viewpoint [type of Angle] & ponto de vista [tipo de Ângulo] \\
\hline Vocative [interpersonal clause function] & Vocativo [função interpessoal da oração] \\
\hline vocative [minor clause] & vocativo [oração menor] \\
\hline voice system & sistema de voz \\
\hline \multicolumn{2}{|c|}{ W } \\
\hline WH-element & elemento Qu- \\
\hline WH-interrogative & interrogativa Qu- \\
\hline wh-selection & seleção Qu- \\
\hline word [rank] & palavra [nível] \\
\hline word group & grupo de palavras \\
\hline wording & fraseado \\
\hline \multicolumn{2}{|c|}{$\mathbf{Y}$} \\
\hline yes/no interrogative & interrogativa sim/não \\
\hline
\end{tabular}




\section{REFERÊNCIAS}

CUNHA, Maria Angélica Furtado da; SOUZA, Maria Medianeira de. Transitividade e seus contextos de uso. São Paulo: Cortez, 2011.

FUZER, Cristiane; CABRAL, Sara Regina Scotta. Introdução à gramática sistêmicofuncional em língua portuguesa. Campinas, SP: Mercado de Letras, 2014.

HALLIDAY, Michael Alexander Kirkwood. An introduction to functional grammar. 2. ed. London: Arnold, 1994.

HALLIDAY, Michael Alexander Kirkwood; MATTHIESSEN, Mathias Ingemar Martin. An introduction to functional grammar. 3. ed. London: Hodder Arnold, 2004.

HALLIDAY, Michael Alexander Kirkwood; MATTHIESSEN, Mathias Ingemar Martin. Halliday's introduction to functional grammar. 4. ed. New York, London: Routledge, 2014.

Submetido em: 15 maio 2021

Aceito para publicação em: 28 maio 2021

DOI: $\underline{\text { http://dx.doi.org/10.22456/2238-8915.114042 }}$ 